\title{
Exploiting Time and User Diversity in Distributed Medium Access Control for Mobile Ad-Hoc Networks
}

\author{
Qinqing Zhang, Senior Member, IEEE, Lotfi Benmohamed, Senior Member, IEEE, I-Jeng Wang, \\ Senior Member, IEEE
}

\begin{abstract}
In this paper, we consider a distributed medium access control (MAC) for mobile ad-hoc networks (MANET). We propose an adaptive threshold-based medium contention scheme to exploit diversity gain in multiple dimensions, in particular, the time and user diversity. In our scheme, each node performs a carrier sensing multiple access with collision avoidance (CSMA/CA) MAC protocol and access the channel only when the channel quality is above a threshold. We develop a practical solution to address node mobility and time varying fading channels in a general ad-hoc network model. Our solution consists of channel estimation and prediction, transmission rate selection, and threshold adaptation. We show that an efficient threshold-based solution will need to employ a dynamic loadbased threshold adaptation. Moreover, we show that a distributed MAC algorithm requires additional overhead for time and user diversity control and thus need appropriate tradeoff design to maximize the system capacity. We conduct network simulations to evaluate the performance of our proposed protocol and demonstrate promising throughput increase via time and user diversity. We conclude that the achievable diversity gain in MANET highly depends on the network topology and heterogeneity of link quality and thus remains as a challenging task.
\end{abstract}

Index Terms-medium access control, mobile ad-hoc networks, time and user diversity, 802.11, distributed algorithms

\section{INTRODUCTION}

Mobile Ad-Hoc Network (MANET) has become an emerging communication technology for commercial and military networks. Different from cellular wireless networks, MANET has a number of unique characteristics including 1)rapid and unpredictable changes in topology and connectivity; 2) lack of a centralized controller, i.e., a decentralized network; 3) multi-hop transmission for a data packet between a source and destination. These main characteristics have brought many research challenges in the

Q. Zhang, L. Benmohamed and I. Wang are with Milton S. Eisenhower Research Center at Johns Hopkins University, Applied Physics Laboratory, Laurel, Maryland, USA. E-mails: qinqing.zhang@jhuapl.edu, lotfi.benmohamed@jhuapl.edu, I-jeng.wang@jhuapl.edu. design of efficient and effective mobile ad-hoc networks to meet the diverse application and deployment requirements.

Many of the resource management algorithms and medium access control (MAC) protocols are designed for a centralized system where an anchor node (such as a base station in cellular systems or access point in wireless LANs) manages and allocates resources to mobile nodes in order to make efficient use of the radio resources. However, in a MANET environment, the lack of a central control entity makes medium access control and scheduling a challenging task. There is a need to design and develop efficient MAC and resource management algorithms in a distributed manner to maximize the network throughput and meet the applicationlevel QoS requirements.

The time varying channel condition in MANET is a significant challenge for network design. Advances in wireless communications have created innovative techniques that take advantages of the time varying channel and mobility to improve the system capacity and performance [1]. One of the key techniques is to explore diversity gain in multiple dimensions, including frequency, spatial, time and user diversity. Exploiting these diversities requires developing appropriate methods and algorithms to gain knowledge of the channel by tracking and predicting the channel quality information. Improving the overall network capacity and performance requires developing appropriate methods and algorithms to gain knowledge of the user specific radio frequency (RF) condition and application traffic requirement. Innovative techniques in radio resource management have been designed for infrastructure based commercial wireless systems and networks [2][3]. In this paper, we propose to develop an intelligent MAC protocol with a design approach that focuses on the development of distributed algorithms to exploit time and user diversity to improve the network efficiency. We propose a novel adaptive threshold-based medium access control protocol that includes channel estimation and prediction, transmission rate selection, and threshold adaptation. Note that channel or frequency diversity, spatial diversity such as MIMO techniques, and multi-path diversity are additional dimensions that can be explored but are not within the scope of this paper.

Over the recent years there has been some work on exploiting multi-user diversity in a distributed manner, in particular [4], [5], and [6]. In [4], the authors deal with 
channel-aware ALOHA using a decentralized MAC protocol where a source node would access the medium only when the channel gain is larger than a threshold. They consider a centralized network model where many nodes communicate with an access point. Each node is only aware of its own channel state but has the same independent Rayleigh fading distribution. In [5], the authors use a similar wireless LAN model (one-hop communication through an access point) and combine power control with threshold-based user diversity control. Different from the above two that are based on singlehop LAN model, in [6], the authors consider ad-hoc communication. However to make the analysis tractable it uses a model based on random access without carrier sensing. In this paper we address the general ad-hoc model based on the widely used carrier sensing multiple access with collision avoidance (CSMA/CA) type of MAC. We develop a practical solution for this realistic model. Our solution is an enhanced and compatible version of the 802.11 MAC standards. We consider the impact of node mobility and time varying fading channel in our design and analysis. Moreover, we show that an efficient threshold-based solution will need to employ a dynamic load-based threshold adaptation. Finally we show that a distributed MAC algorithm requires additional overhead for time and user diversity control, and thus need proper consideration of tradeoff in the design.

This paper discusses the design and analysis of a MAC protocol that allows exploring diversity in multiple dimensions, in particular the time and user diversity, thus resulting in additional diversity gain. Section 2 defines the basic system model that we address in this paper. Section 3 describes in detail our proposed distributed MAC protocol. Section 4 provides performance analysis results from simulation studies. Section 5 concludes the paper and discusses some open issues.

\section{SYSTEM MODEL}

We assume a mobile ad-hoc system architecture where wireless nodes are configured without any centralized entity that controls medium access and transmission. Each node acts as a router, forwarding data packets to other nodes in the network. A data packet may reach its destination through multiple replaying nodes, i.e., multi-hop transmission.

We consider the widely used CSMA/CA type of MAC as the basis of our MAC protocol design. Particularly, we are interested in the 802.11 standard MAC with the request to send (RTS) and clear to send (CTS) option, to reduce frame collision introduced by the hidden terminal problem and exposed terminal problem. In the RTS/CTS mechanism, when a node has data to send, it first sends a RTS frame to the nexthop neighboring node to request reservation of the channel. Upon receipt of the RTS frame, the next-hop neighboring node replies with a CTS frame for confirmation. Upon receipt of the CTS frame, the sender node sends the data frame with the reserved time duration. Any other nodes receiving the CTS frame will wait for the sending node to finish the data frame transmission before trying to access the medium. Any other node receiving the RTS frame but not the CTS frame is permitted to send data to other neighboring nodes as it is not within radio range of the receiver and hence would not interfere with the sender's transmission.

The diversity gain is highly dependent on the characteristics of the wireless channels and the effectiveness of channel estimation and prediction. In a rough RF environment, the wireless channel profile can be dramatically different from link to link. The transmitter needs to gain knowledge of the channel and track the variation effectively. This is usually achieved by channel estimation and feedback information from the receiver. We develop a receiver-based channel estimation method to track and predict the channel quality. We use the RTS/CTS handshake mechanism to exchange the information about channel state and adjust the transmission rate.

We consider a Rayleigh fading model with slow moving speed of $8 \mathrm{~km}$ per hour and examine how much diversity gain can potentially be achieved. The slow moving speed results in a large channel coherence time, which makes the channel estimation easier to implement. With fast moving speed, the channel estimation and prediction becomes much more difficult. The diversity gain is expected to be smaller.

Multiple transmission rates are supported in both wireless LAN and 3G/4G wireless systems to adapt to the varying link quality. Link adaptation improves the spectrum efficiency and increases the system capacity. We use the multiple transmission rate set in $802.11 \mathrm{~b}$ standards. Six different transmission rates are used in our simulation studies. The rate is selected based on the estimated signal to interference plus noise ratio (SINR) at the receiving node. The receiving node sends the rate indication along with the CTS frame to the transmitting node.

Exploring time diversity in a fading channel and performing link adaptation accordingly provides an effective way of mitigating the fading impairment. To maximize the capacity and throughput at the system level, user diversity needs to be considered as well. The notion of exploring user diversity means that for a shared medium such as a wireless channel, the channel reservation and assignment is given to the user who has the best channel quality compared to other users. This can be done in a relatively easy way in an infrastructure mode where a centralized entity is aware of each user's channel condition. In a decentralized network, a distributed algorithm and protocol is needed for cooperation and collaboration among the nodes to optimize the system capacity. The following section provides the details of our distributed implementation.

\section{DISTRIBUTED CSMA MAC PROTOCOL WITH MULTI- DIMENSIONAL DIVERSITY}

In this section, we describe a novel distributed MAC protocol to exploit both time and user diversity in a MANET environment. The main design consists of the following components.

\section{A. Channel estimation and transmission rate selection}

We develop a receiver-based channel estimation and prediction method to track the variation of a wireless fading channel over time. In MANET, since each mobile node may 
receive packets from all of its neighboring nodes, a node needs to maintain the channel state information from each neighbor and update its neighbor list periodically as the network topology changes. The receiving node estimates the channel quality by measuring the received signal to interference-plus-noise ratio (SINR) of the received frames from the sender. The received frame can be either a data packet or a control packet. Each node updates its estimate of the channel quality when receiving a frame at time $t$ from sender $k$. The information about channel quality from sender $k$, represented by the SINR value, is updated by the receiver at time $t$ as follows:

$$
\hat{\gamma}_{t}^{k}=\frac{\beta}{\beta+(1-\beta)^{t-t_{0}}} \gamma_{t}^{k}+\frac{(1-\beta)^{t-t_{0}}}{\beta+(1-\beta)^{t-t_{0}}} \hat{\gamma}_{t_{0}}^{k}, t_{0}<t, \beta \in[0,1] \text { (1) }
$$

where $\hat{\gamma}_{t}^{k}$ is the estimated SINR value at time $t ; \gamma_{t}^{k}$ is the measured received SINR value at time $t ; \hat{\gamma}_{t_{0}}^{k}$ is the estimated SINR value at time $t_{0}$, the last time such an update took place, and $\beta$ is a constant parameter of the moving average estimator. The choice of $\beta$ depends on how fast the channel changes over time. Both time $t$ and $t_{0}$ are discrete time integers.

The receiving node sends a channel state indication to the transmitting node. The channel state indication can be either a direct rate selection or the actual SINR value for the transmitting node to select the corresponding transmission rate. We implemented the former in our simulation described in the next section. The receiving node selects a transmission rate based on the SINR estimate value and sends it back to the transmitting node. The SINR to rate selection is derived from the performance data sheet of a commercial WLAN chip product [7].

\section{B. Adaptive threshold based medium contention}

We propose an adaptive threshold based contention scheme to exploit both the time and user diversity gain in the shared wireless medium. When a mobile node has data to send, it sends a RTS frame to the receiving node for channel reservation. Upon receipt of a CTS frame with the transmission rate indication from the receiving node, it compares the rate with a threshold. If the indicated rate is larger than the threshold, it sends the data frame. Otherwise, the node aborts the data transmission and waits for the next contention after a back off time period.

We consider two different methods of setting the threshold at each mobile node. The first method is to use a common threshold setting for all the nodes in the network. The common threshold value is set to maximize the aggregate throughput of the network. In this method, only nodes whose channel condition is above a rate threshold are allowed to send. Nodes whose channel condition is worse than the threshold will not be able to transmit data. This method will give more transmission opportunities to users with better channel quality, while penalizing users with poor channel quality. As a result, it will achieve a higher throughput. Obviously, the drawback of this method is that it does not provide any fairness among the users. Users who are in poor RF conditions will be prevented for transmission if the achievable transmission rate is below the threshold.

We also consider a second method of setting the threshold to maximize the network throughput and also achieve fairness among the users. Since each node may transmit data to different neighboring nodes, a node maintains its own threshold associated with each of its neighboring nodes and adapts the value dynamically. The threshold, represented by a transmission rate, is adjusted when receiving a rate indication from node $j$ as

$$
T_{\text {new }}^{j}=\rho \cdot\left(\alpha R^{j}+(1-\alpha) T_{\text {old }}^{j}\right) \alpha, \rho \in[0,1]
$$

where $T_{\text {new }}^{j}$ is the updated threshold to receiving node $j$; $T_{\text {old }}^{j}$ is the previous threshold value; $R^{j}$ is the transmission rate indication from receiving node $j ; \alpha$ is a constant of the moving average of the threshold; $\rho$ is a loading coefficient, which is set dynamically based on the channel utilization estimation. For simplicity, we set $\rho$ as an increasing function of the observed channel busy time percentage at the node,

$$
\rho=f\left(\frac{D_{\text {busy }}}{D_{\text {observation }}}\right)
$$

where $D_{\text {observation }}$ is the observation time duration; $D_{\text {busy }}$ is the duration of the medium usage by other nodes during $D_{\text {observation }}$; and $f(\cdot)$ is a monotonic increasing function of the input variable. The coefficient $\rho$ increases as the channel busy time increases. The motivation of using the loading coefficient $\rho$ in the threshold update in Eq. (2) is to make the threshold dynamically adapted to the loading. When the load is high with many nodes and flows accessing the medium, the threshold is scaled to a higher value. A higher threshold will force the node to back off more often and release the channel for other nodes to use. This will lead to an increased system capacity with more user diversity gain. On the other hand, when the load is low without many nodes or flows utilizing the medium, the user diversity gain will be limited. A smaller threshold value will decrease the intentional back off and thus helps in reducing the packet delay.

\section{Channel probing and estimation overhead}

Channel estimation is critical in tracking the time varying fading channel and improving the transmission efficiency. However, channel probing and estimation in a distributed system introduces additional overhead, which should be considered carefully in the protocol design. In our MAC protocol, we use the RTS and CTS handshake mechanism to probe the channel and obtain the channel state indication. Only when the indicated transmission rate is above a threshold, the node will send the data frame. Therefore, for a node to actually transmit a data frame, it may need multiple RTS and CTS handshakes to probe the channel and find the right time to transmit a data frame. The achieved time diversity gain (in perspective of individual user) as a result of higher transmission rate has a cost of additional overhead. The 
achieved user diversity gain as a result of another node transmitting a data frame at a higher rate has the same cost of overhead. This additional overhead will hamper the user and time diversity gain and cost the system throughput eventually. The threshold selection should be designed carefully to achieve the best tradeoff between the overhead and potential diversity gain to optimize the system capacity.

\section{Summary of our proposed distributed MAC protocol}

Our proposed distributed MAC protocol is operated as follows.

o Each node maintains channel state information of each of its neighboring nodes, according to equation (1).

o Each node maintains a rate threshold associated with each of its neighboring nodes.

o When a node has data to send, it sends a RTS frame to reserve the channel.

o The receiving node uses the channel state information to select the transmission rate. It sends back a CTS frame with the transmission rate indication $R^{j}$.

0 The sender receives the rate indication and compares it with the threshold. If $R^{j}<T_{\text {old }}^{j}$, the node aborts the data frame transmission and sends a RES frame to all the neighboring nodes. The node backs off waiting for the next contention. Otherwise, the node sends the data frame using rate $R^{j}$.

o The sender updates its threshold according to (2) and (3).

In the next section, we evaluate the performance of our proposed MAC protocol and compare it with the standards 802.11 MAC protocol.

\section{Performance eVAluation}

\section{A. Simulation Descriptions}

We developed a network simulation model using the GlomoSim simulation tool [9] to evaluate the performance of our proposed MAC protocol. The simulation assumptions and parameters are described as follows.

\section{1) Network topology and routing}

We place different number of nodes uniformly in a $600 \times 600$ meters terrain space. We vary the number of application flows among the nodes. The flow's source and destination is randomly selected among the nodes. Static routes are used to communicate between the nodes. Shortest path routing is used to configure the static routing table used in the simulation. Each flow's transmission can be a single hop or multi-hop depending on the route selected for the source and destination. In most of our simulation scenarios, at most two-hop transmission is needed for a flow.

\section{2) Application traffic}

We configure each flow as an application in GlomoSim settings. The FTP GENERIC application traffic generator, which does not use the TCP library to generate any control data, is used for each flow. We configure the traffic generator to let the client node continue sending a fixed size application layer packet to the server node. The packet size is set as 1400 Bytes. This traffic generator represents a backlogged traffic model, a scenario for saturated system capacity.

\section{3) Path loss and fading model}

We use the two-ray path loss propagation model defined in GlomoSim. The radio configuration parameters are listed in Table I.

TABLE I RADIO CONFIGURATION PARAMETERS

\begin{tabular}{|l|l|}
\hline RADIO-TX-POWER & $13 \mathrm{dBm}$ or $7 \mathrm{dBm}$ \\
\hline RADIO-ANTENNA-GAIN & $0.0 \mathrm{dBm}$ \\
\hline RADIO-RX-SENSITIVITY & $-91 \mathrm{dBm}$ \\
\hline RADIO-RX-THRESHOLD & $-88 \mathrm{dBm}$ \\
\hline RADIO-RX-TYPE & BER-BASED \\
\hline
\end{tabular}

We model the wireless fading channel using the traditional Jakes' model [8] to generate a time series of samples of the channel gain. The sample interval is $1 \mathrm{~ms}$. A slow moving speed of $8 \mathrm{~km}$ per hour is used in the Jakes' model to generate the fading coefficient. The fading channels between each pair of nodes are assumed to be independent.

\section{4) Rate adaptation}

We follow the multiple transmission rate set used in the 802.11b standards and interpolate two additional rates. The transmission rates are 1, 2, 5.5, 8, 9.5, and 11 Mbps. We use the BER-based parameter and look up bit-error-rate (BER) in the SINR-BER table derived from a commercial WLAN chip product [7].

\section{5) Threshold-based MAC protocol parameters}

The parameters used in our proposed threshold-based MAC protocol are summarized in Table II.

TABLE II THRESHOLD-BASED MAC PROTOCOL PARAMETERS

\begin{tabular}{|l|l|}
\hline$\beta$ in channel estimation, Eq (1) & 0.75 \\
\hline$\alpha$ in threshold adaptation, Eq. (2) & 0.5 \\
\hline Loading $\rho$, Eq. (3) & $=1$, if channel busy $>50 \%$ \\
& $=0.5$, else if channel busy $>20 \%$ \\
$=0.2$, else \\
\hline Common Threshold & 5.5 Mbps, or 8 Mbps \\
Adaptive Threshold & Eq. (2) and (3) \\
\hline
\end{tabular}

\section{B. Simulation results}

We conducted intensive simulations to evaluate and compare our proposed MAC protocol with the standard 802.11 MAC protocol [10]. We vary the number of flows in the simulations to examine the achievable time and user diversity gain using our distributed MAC protocol.

Figure 1 shows the average transmission rate versus number of flows. The average transmission rate is measured as the selected rate for transmitting a data frame average over all the data frames among the nodes, excluding the rates used for the control frames. We also compare the average transmission rate when using two threshold selection methods, i.e., the common threshold and the adaptive threshold method. We can see that the average transmission rate for data frame increases when 
using a threshold to determine the transmission. With a common threshold of $5.5 \mathrm{Mbps}$ (one of the transmission rate is the discrete rate set), the average transmission rate has an increase as much as $57 \%$. With the adaptive threshold method, the average transmission rate has an increase about $35 \%$. In this simulation scenario, the average transmission rate with no threshold case is around 3500 kbps. Since the highest transmission rate is $11 \mathrm{Mbps}$ in $802.11 \mathrm{~b}$ rate set, there is a large room for transmission rate increase when properly setting the threshold to allow each node to transmit at its good channel condition. This set of results indicates the potential time diversity gain when using a threshold to control the transmission.

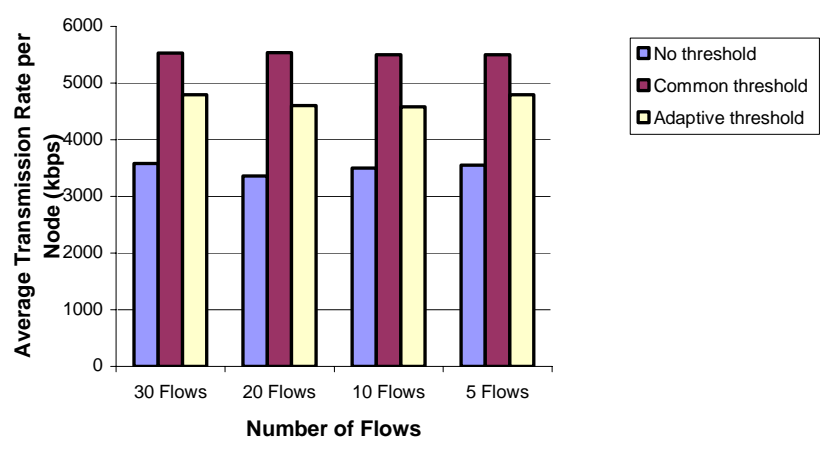

Figure 1 Comparison of Average Transmission Rate

Figure 2 shows the average transmission rate versus number of flows when nodes are in better RF conditions. In this scenario, the transmission power at the nodes is set at a higher value $(13 \mathrm{dBm}$ instead of $7 \mathrm{dBm})$. The average transmission rate with no threshold case is around $6600 \mathrm{Mbps}$. We can see that there is still potential increase in transmission rate with a distributed threshold setting. With a common threshold of 8 Mbps, the average transmission rate has an increase as much as $30 \%$. With the adaptive threshold method, the average transmission rate has an increase of $18 \%$. The time diversity gain has a lower quantitative gain than that in Figure 1. This is due to the fact of using the discrete rate set in $802.11 \mathrm{~b}$, which has a maximum of 11 Mbps transmission rate. Thus the time diversity gain is limited by this cap. If using other advanced physical layer design which allows a higher transmission rate, the time diversity gain is expected to be higher than the value shown here.

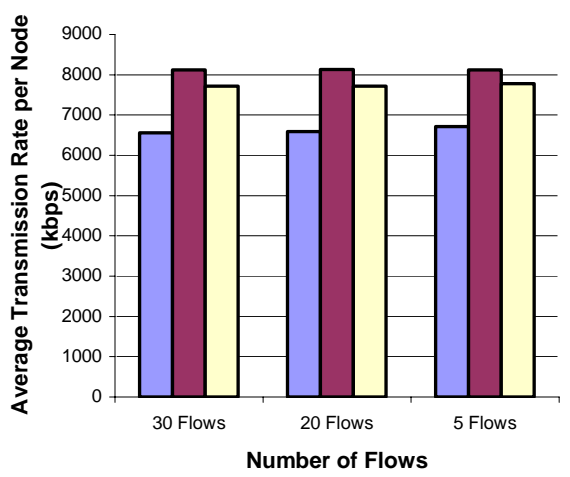

QNo threshold

口Common threshold

口Adaptive threshold

Figure 2 Comparison of Average Transmission Rate (nodes with better RF conditions)

Figure 3 shows the aggregate system throughput with 16 nodes versus the number of flows. The aggregate throughput is measured as the total transmitted bits at the MAC layer, average over the simulation time, excluding the extra control frames (due to RTS frame collision) transmission time. We can see that the system throughput increases with our distributed MAC protocol. With 20 flows, the system throughput increases about 20\%. Compared to the transmission rate, the increase is less than that shown in Figure 1. The reason is because of the additional overhead in exploiting the channel condition, which reduces the gain in system throughput. With 5 flows, the system throughput actually decreases compared with the no threshold case. This is because that although the individual data frame transmission rate is increased, the additional channel probing (RTS/CTS handshakes) actually result in longer time for transmitting a data frame. In addition, because the number of flows is small, the potential user diversity gain is very limited. In other words, when a node backs off due to poor channel condition, there is no other nodes with data available to transmit to utilize the channel.
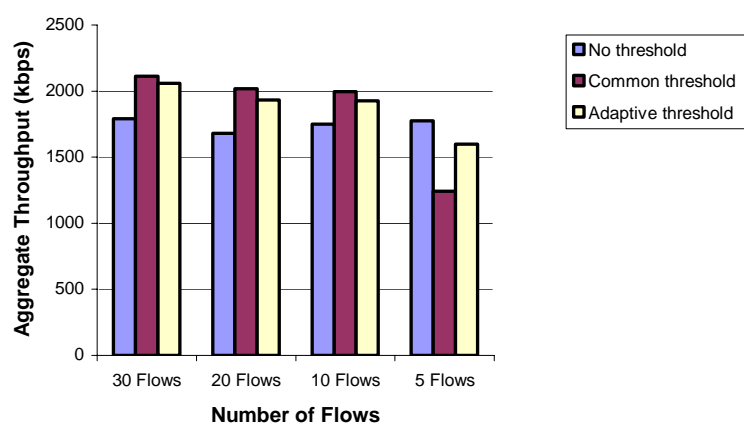

口Adaptive threshold

Figure 3 Comparison of Aggregate System Throughput

Figure 4 shows the average throughput per node distribution, with 10 flows among the 16 nodes. Only nodes with active flows are plotted in the figure. We can see that with no threshold case, the throughput per node distribution varies 
based on the channel condition. Each node gets roughly the same amount of transmissions to access the medium. With the common threshold method, nodes with better channel quality access the medium more often than nodes with poor channel conditions. In fact, in the simulation scenario of 10 flows, two nodes are completely blocked from transmission due to the high threshold value. On the other hand, with the adaptive threshold method, each node gets approximately the same chance to access the medium. The adaptive threshold method achieves better fairness of transmission among the nodes, at a slightly lower transmission rate and aggregate throughput as shown in Figure 1 and Figure 3.

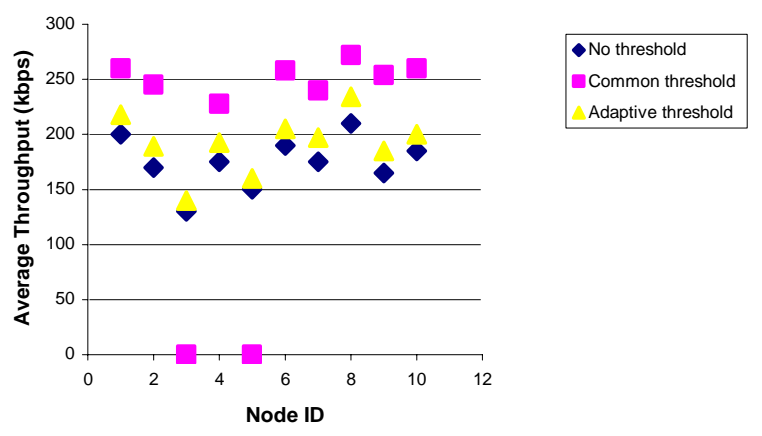

Figure 4 Fairness Comparison of Average Throughput per Node

\section{CONCLUSION}

In this paper, we developed a distributed MAC protocol for mobile ad-hoc networks where there is no centralized controlling entity to coordinate the access and transmission over the shared wireless medium. We proposed an adaptive threshold-based contention scheme to exploit time and user diversity gain over a time varying fading channel. Each mobile node would access the channel only when the channel quality is above a threshold. We demonstrated that time and user diversity gain could be achieved in this distributed protocol where each node adapts and contents for the channel without any coordination among them. We developed network simulations for performance evaluation and showed promising results on the achievable diversity gain in a practical system.

It is interesting to notice that the performance improvement via multi-dimensional diversity gain highly depends on the network topology and the link quality variation. In a highly dynamic MANET environment with heterogeneous link qualities, achieving potential user diversity gain is a challenging task. We observed that in some network topology scenarios, the diversity gain is very minimum. The overall achievable diversity gain in MANET is smaller than the gain in centralized networks. More sensitivity analysis on topology and deployment scenarios will be reported in our future work.

\section{ACKNOWLEDGMENT}

The authors would like to thank Ashwin Kowdle and Junshan Zhang from Arizona State University for developing the network simulator in Glomosim for their initial investigation on multi-channel and MIMO MAC protocol design.

\section{REFERENCES}

[1] M. Grossglauser and D. Tse, "Mobility Increases the Capacity of AdHoc Wireless Networks," Proceedings of IEEE INFOCOM 2001, pp. 1360-1369, 2001.

[2] M. Andrews, K. Kumaran, K. Ramanan, A. Stolyar, P. Whiting, and R. Vijayakumar, "Providing Quality of Service over a Shared Wireless Link,” IEEE Communication Magazine, No. 39, pp. 150-154, 2001.

[3] P. Chen, R. Da, C. Mooney, Y. Yang, Q. Zhang, L. Zhu, J. Zou, "Quality of Service Support in 1xEV-DO Revision A Systems," Bell Labs Technical Journal, vol. 11, no. 4, pp. 169-184, 2007.

[4] X. Qin, R. Berry, "Exploiting Multiuser Diversity for Medium Access Control in Wireless Networks," Proceedings of IEEE INFOCOM 2003, pp. 1084-1094.

[5] C. S. Hwang, K. Seong, and J. M. Cioffi, "Opportunistic p-persistent CSMA in Wireless Networks," Proceedings of IEEE ICC 2006, June 2006.

[6] D. Zheng, W. Ge and J. Zhang, "Distributed Opportunistic Scheduling For Ad-Hoc Communications: An Optimal Stopping Approach,” ACM MobiHoc'07, Montreal, Quebec, Canada, 2007.

[7] Intersil Data Sheet, "Direct Sequence Spread Spectrum Baseband Processor,” HFA3861B, February 2002.

[8] W. Jakes, Microwave Mobile Communications, IEEE CRC Press, 1994.

[9] L. Bajaj, M. Takai, R. Ahuja, K. Tang, R. Bagrodia, and M. Gerla, "Glomosim: A Scalable Network Simulation Environment," Technical Report, UCLA Computer Science Department - 990027

[10] IEEE 802.11: Wireless LAN Medium Access Control (MAC) and Physical Layer Specifications, 1998. 